\title{
Effect of Low-Intensity Pulsed Ultrasound on Quality of Life of Knee Osteoarthritis
}

\author{
Joudy Gessal $^{1}$, Irawan Yusuf ${ }^{2}$, Ilhamjaya Patellongi ${ }^{2}$, Gatot Lawrence ${ }^{3}$ \\ ${ }^{1}$ Department of Physical Medicine and Rehabilitation, Faculty of Medicine, Sam Ratulangi University, Manado, Indonesia \\ ${ }^{2}$ Department of Physiology, Faculty of Medicine, Hasanuddin University, Makassar, Indonesia \\ ${ }^{3}$ Department of Pathology Anatomy, Faculty of Medicine, Hasanuddin University, Makassar, Indonesia \\ Email address: \\ jgessal@yahoo.com (J. Gessal), irawanyusufgenome@gmail.com (I. Yusuf), ilham_pt@yahoo.com (I. Patellongi), \\ gatot.law@gmail.com (G. Lawrence)
}

\section{To cite this article:}

Joudy Gessal, Irawan Yusuf, Ilhamjaya Patellongi, Gatot Lawrence. Effect of Low-Intensity Pulsed Ultrasound on Quality of Life of Knee Osteoarthritis. American Journal of Health Research. Vol. 3, No. 3, 2015, pp. 166-169. doi: 10.11648/j.ajhr.20150303.20

\begin{abstract}
Background: Knee osteoarthritis (OA) is a common disease worldwide and causes disability in elderly. Recent studies showed that therapeutic ultrasound (US) can reduced pain in various musculoskeletal disorders. Objective: To determined the effect of low-intensity pulsed US on quality of life and reduction of pain in grade 1 to 3 knee OA. Material and methods: We conducted a randomized control trial before and after therapy in 61 knee OA patients. We applied 12 sessions of low-intensity pulsed US, 20 minutes each, with frequency $1 \mathrm{MHz}$, and intensity of $0.2 \mathrm{~W} / \mathrm{cm} 2$, duty cycle of $20 \%$ to 28 patients. Thirty three patients without therapy were categorized as control group. Reduction of pain by a visual analog scale and Quality of life by The Western Ontario and McMaster Universities (WOMAC) questionnaire, were obtained before and after 12 sessions. We used the Wilcoxon test to compare the median and the Mann-Whitney U test for the difference between groups with a $\mathrm{p}$ value of 0.05 was considering as significant. Results: We observed a reduction of pain in therapy group (VAS before $3.5(2 / 7)$; after $2.0(0 / 5) ; p=0.001)$ and also in control group (VAS before 5.1 (2/8); after 4.0 (2/7); p=0.001), but the reduction was significantly more prominent in therapy group $(\mathrm{p}=0.004)$. There was a better quality of life in therapy group (WOMAC before $21.35(4.2 / 52.1)$; after $11.45(0.0 / 47.9) ; \mathrm{p}=0.001$ ) and also in control group (WOMAC before 25.00 $(5.2 / 60.4)$; after $20.83(3.1 / 55.2) ; p=0.001)$, but the score was significantly better in therapy group ( $\mathrm{p}=0.001)$. Conclusions: Twelve sessions of low-intensity pulsed US has a benefit effect over pain and quality of life in knee OA patients.
\end{abstract}

Keywords: Low-Intensity Pulsed Ultrasound, Knee Osteoarthritis, Quality of Life, VAS, WOMAC

\section{Introduction}

Osteoarthritis (OA) is a common disease associated with significant morbidity, and its prevalence increases with age [1]. Knee OA is the most common presentation of OA, with an estimated prevalence between $12 \%$ and $35 \%$ in the general population and is considered the leading cause of musculoskeletal disability in the elderly population worldwide. Osteoarthritis which result in the deterioration of joint structure and function is one of the cause of long term disability and pain in adults. Functional deficit, pain and stiffness of the patient with severe OA may have influence on the quality of life and had a significant economy impact [2].

Ultrasound therapy is one of the most widely used physical agents in physiotherapy practice. This treatment modality is based on the application of mechanical energy, produced by sound waves at different frequencies, to a tissue in order to induce a biological response [3]. Therapeutic US treatment, such as those using low-intensity pulsed US wave energy, are widely used to treat pain and various musculoskeletal disorders including bone fractures, shoulder pain, pressure ulcers, and muscle soreness [4,5]. Recent clinical trials show therapeutic US such as low-intensity US can improve OAassociated pain and dysfunction, although its effects in modifying disease progression require to be further studied [6]. The other study had shown that the mechanical stimulus produced by low-intensity ultrasonic energy (spatial average temporal average intensities between .03-.62W/cm2) promotes chondrogenesis in human cell cultures and animal models of cartilage injury. These observations suggest that low-intensity pulsed US could favor the repair of injured 
cartilage and, if applied at early stages, may slow the progression of OA. The mode of action is unclear, although exciting new studies indicate that US activates the osteoblast function [7]. Other study suggest that US may stimulate bone healing processes such as endochondral ossification, and inducing collagen and glycosaminoglycan production [8]. Consequently, low-intensity pulsed US may also be beneficial for cartilage regeneration either in vivo or as part of an in vitro tissue engineered approach [9].

The Western Ontario and McMaster Universities (WOMAC) questionnaire measure health related quality of life, developed for OA in the hip and in the knee [10,11]. Visual analogue scales (VAS) are often used in epidemiologic and clinical research to measure the intensity or frequency of various symptoms, particularly pain. They are generally completed by patients themselves but are sometimes used to elicit opinions from health professionals [12]. The aim of this study was to investigate the effect of low-intensity pulsed US on quality of life and reduction of pain in grade 1 to 3 knee osteoarthritis (OA) patients.

\section{Subject and Methods}

We conducted a randomized control trial before and after therapy in R.D. Kandou Hospital, Manado, Indonesia, since May until October 2014. Sixty one patients aged 33 to 77 years, with knee OA were included in this study. A simplecomputerized random-number generator was used to allocate the participants to 1 of 2 groups (therapy or control). The diagnosis of knee OA according to the criteria of American College of Rheumatology [13], stages 1, 2 and 3 according to the classification by Kellgren-Lawrence (KL) [14]. Exclusion criterias were OA stage $4 \mathrm{KL}$, intra articular knee effusion, contraindication for using US, corticosteroid intra articular injection 6 months prior to the study. Drop out criteria was patient not continue all session. This study was approved by the ethics committee of Kandou Hospital, Manado, Indonesia

We applied 12 sessions of low-intensity pulsed US, 20 minutes each, with frequency $1 \mathrm{MHz}$, and intensity of 0.2 $\mathrm{W} / \mathrm{cm} 2$, duty cycle of $20 \%$ using an intellect mobile model physiomed therapeutic US apparatus. Application of lowintensity pulsed US was performed by physiatrist, in the Department of Physical Medicine and Rehabilitation, Kandou Hospital. Participants were positioned in a supine position with the affected knee flexed at $90^{\circ}$ and the soundhead was held stationary over the tibiofemoral joint superomedial and lateral to the patellar tendon to enhance energy penetration into the joint space. Twenty eight patient received treatment were categorized as therapy group and 33 patients without therapy were categorized as control group. The severity of knee pain was assessed on 2 occasion, before the start of treatment and after 12 sessions using a visual analog scale (VAS) consisting of a $10 \mathrm{~cm}$ horizontal line, with anchor points of 0 (no pain) and 10 (maximum pain). Quality of life was obtained by The Western Ontario and McMaster Universities (WOMAC) questionnaire which has been validated in the knee OA population, at baseline and after the completion of the interventions. This 24-item questionnaire has 3 domains (pain, stiffness, and physical function) and the total score ranges from 0 (best) to 96 (worst).

We used the Wilcoxon test to compare the median and the Mann-Whitney $U$ test for the difference between groups with a $\mathrm{p}$ value of 0.05 was considering as significant.

\section{Results}

A group of 61 patients were studied, formed by 28 patients of therapy group and 33 patients of control group. The mean age of therapy group was 56.3 (10.6) years and control group was $56.8(11.2)$ years. The median of OA KL stages in both group were $3(1 / 3)$. Demography characteristics of 2 groups before therapy were showed in table 1 and clinical characteristics were showed in table 2 .

Table 1. Baseline demographic characteristics.

\begin{tabular}{lll}
\hline Variable & Therapy $(\mathbf{n}=\mathbf{2 8})$ & Control $(\mathbf{n}=\mathbf{3 3})$ \\
\hline Age, mean $(\mathrm{SD})$, years & $56.3(10.6)$ & $56.8(11.2)$ \\
BMI, mean (SD), Kg/cm2 & $25.53(3.21)$ & $25.77(3.23)$ \\
Sex women, n (\%) & $24(85.7)$ & $29(87.9)$ \\
\hline
\end{tabular}

Joint pain measured by Visual Analog Scale showed a significant reduction in therapy group (mean VAS before 3.7 (1.3); after 2.1 (1.2); $\mathrm{p}=0.001$ ) and also in control group (VAS before 4.9 (1.5); after $3.9(1.4)$; $\mathrm{p}=0.001)$. There was a significant difference between the reduction of therapy group and control $(\mathrm{p}=0.004)$.

There was a better quality of life measured by WOMAC in therapy group (WOMAC before 24.76 (12.37); after 13.46 (11.01); $\mathrm{p}=0.001$ ) and also in control group (WOMAC before 29.88 (14.76); after 24.47 (13.19); $\mathrm{p}=0.001)$, There was a significant difference the increase quality of life between therapy group and control $(\mathrm{p}=0.001)$.

Table 2. Baseline clinical characteristics.

\begin{tabular}{lll}
\hline Variable & Therapy $(\mathbf{n}=\mathbf{2 8})$ & Control $(\mathbf{n}=\mathbf{3 3})$ \\
\hline $\begin{array}{l}\text { Visual analogue scale, median } \\
(\min / \max )\end{array}$ & $3.5(2 / 7)$ & $5.1(2 / 8)$ \\
WOMAC, median (min/max) & $21.35(4.2 / 52.1)$ & $25.00(5.2 / 60.4)$ \\
\hline
\end{tabular}

\section{Discussion}

Osteoarthritis is a joint disorder that can be caused by a joint wear or tear and the causes are not fully understood yet, however, it is mainly due to aging, genetic inheritance, obesity, long-term overuse and fractures and its typical symptoms include pain, stiffness, cracking sound, and swelling of the joint. Articular cartilage is a firm and rubbery connective tissue found in several areas in the human body especially in a joint and intervertebral disc. It functions as cushion for the bone to bone and it allows gliding over. Since there is no blood supply in articular cartilage, it causes the arrested the repairing process when the articular cartilage is 
injured [15]. Although several treatment methods are available in clinics such as direct surgery, medications and physical therapy, those methods are still in development and cannot provide full recovery of injured cartilage so far.

Ultrasound therapy is one of the most common physical agents used within physiotherapy practice in several countries. Ultrasound therapy is based on the application of high frequency sound waves to the tissues of the body in order to obtain mechanical or thermal effects. These effects aim to enhance soft tissue healing, decrease the inflammatory response, increase blood flow, increase metabolic activity, and decrease pain. Moreover, there is some evidence that ultrasonic energy stimulates the repair of joint cartilage in animal models of cartilage injury. Therefore, US could be an effective intervention in the management of pain and disability in people with knee OA [4,6].

In this study, we conducted a randomized, controlled trial to evaluate the effectiveness of low-intensity pulsed US treatments in patients with symptomatic knee OA. Significant decreases were observed in all groups with regard to reduced pain; however, patients in the therapy group demonstrated more improvements significantly compared with the control group. The same result was reported by Tascioglu et al. [16] that found reduction of VAS in therapy and control group, but more prominent in therapy group.

The present study also found significant improvements in total WOMAC scores in both groups compared with baseline, although more improvement significantly demonstrated by the patients in the therapy group

Improvement in pain and quality of life were also observed in the control group in this study. This non-specific treatment effect, often known as the placebo effect, may be attributed to the attention, interest and concern displayed by the physician and to patients' expectations of the effects of treatment [17]. There is also evidence that placebo-induced analgesia is mediated by the release of endogenous opioid peptides [18].

Ozgonenel et al, [19], investigated the effectiveness of US therapy in knee OA, suggested that therapeutic US is an effective treatment modality in knee OA. Loyola-Sanchez, et al. [6] reported that US therapy could have a beneficial effect on pain and physical function in patients with knee OA.

The exact mechanism of action of therapeutic US remains unknown although it is used to treat various musculoskeletal disorders. Depending on the energy and way the US is delivered, the biophysical effects of US are traditionally separated into thermal and non-thermal effects. Thermal effects are caused by vibration or rotation of macromolecules in the tissue, which result in frictional heat and a rise in temperature. Non-thermal effects are characterized by the formation of tiny gas bubbles (stable cavitation) and the movement of liquid around the vibrating bubbles (acoustic streaming) in the tissue. Heat increases are predominately observed in tissues exposed to continuous high intensity US. In tissues treated with low-intensity pulsed US, the nonthermal effects are dominant [3,9]. Analgesia induced by therapeutic US may be the result of increased capillary permeability and tissue metabolism, enhancement of fibrous tissue extensibility and elevation of the pain threshold by thermal mechanisms. Deep heating with US can produce a temporary increase in the extensibility of highly collagenous structures such as tendons, ligaments and joint capsules. Non thermal effects are less well understood and include molecular vibration, which increases cell membrane permeability and thereby enhances metabolic product transport, fibroblast production, collagen synthesis, and alterations to the extracellular matrix arrangement. It is thought that pain relief may occur as a result of the activation of $A-\alpha$ and A- $\beta$ mechanoreceptors that inhibit nociceptive transmission in A- $\delta$ and C-fiber pathways as a proposed paingating mechanism [20].

Loyola-Sánchez et al. [7] assessed the effect of US therapy on cartilage repair by MRI-based techniques in patients with mild to moderate knee OA. In that study, the medial tibia cartilage thickening observed in the experimental group suggests that US may have a positive effect on the cartilage repair process in people with mild to moderate knee OA with age as an important mediator. This possible effect can be explained by the mechano-transduction pathway theory, which suggests that repetitive mechanical stimuli induce extracellular protein production, improving the formation of cartilage [21]. Several studies conducted in animal models of cartilage injury have demonstrated a positive effect of lowintensity US on cartilage repair [22-24].

There were some limitations of the present study. First, we only evaluate short-term effectiveness of therapeutic US. Secondly, the dosage and the intensity of US, the size of the area treated or the duration of the treatment were not addressed in this study. This limits our understanding about the ideal US therapy prescription and there is little in the literature giving guidance on what may be the optimal dosage.

\section{Conclusion}

In conclusion, we suggest that low-intensity pulsed US therapy is a safe and effective treatment modality for pain relief and that it improves quality of life in patients with knee OA. Further research is required to investigate the long-term efficacy, other dosages and forms of application of therapeutic US.

\section{References}

[1] Felson, D.T. Osteoarthritis. In: Fauci, A.S.; Kasper, D.L.; Longo, D.L.; Braunwald, E.; Hauser, S.L. and Jameson, J.L. (eds). Harrison's Rheumatology. 2nd ed. 2010. New York. The McGraw-Hill Companies.

[2] Heidari, B. Knee osteoarthritis prevalence, risk factors, pathogenesis and features: Part I. Caspian J Intern Med. 2011; 2(2): 205-12.

[3] Basford, J.R. and Baxter, G.D. Therapeutic Physical Agents. In: De Lisa, J.A. and Frontera, W.R. (eds). De Lisa's Physical Medicine \& Rehabilitation Principles and Practice. 5th ed. 2010. Philadelphia. Lippincott Williams \& Wilkins. 
[4] Speed, C.A. Therapeutic ultrasound in soft tissue lesions. Rheumatology. 2001;40:1331-6.

[5] Voigt, J.; Wendelken, M.; Driver, V. and Alvarez, O.M. LowFrequency Ultrasound (20-40 kHz) as an Adjunctive Therapy for Chronic Wound Healing. A Systematic Review of the Literature and Meta-Analysis of Eight Randomized Controlled Trials. International Journal of Lower Extremity Wounds. 2011;10(4):190-9

[6] Loyola-Sanchez, A.; Richardson, J. and MacIntyre, N.J. Efficacy of ultrasound therapy for the management of knee osteoarthritis: a systematic review with meta-analysis. Osteoarthritis Cartilage. 2010. 18:1117-26.

[7] Loyola-Sánchez, A.; Richardson, J.; Beattie, K.A.; OteroFuentes, C.; Adachi, J.D. and Mac Intyre, N.J. Effect of lowintensity pulsed ultrasound on the cartilage repair in people with mild to moderate knee osteoarthritis: a double-blinded, randomized, placebo-controlled pilot study. Arch Phys Med Rehabil. 2012.93:35-42.

[8] Bohari, S.P.M; Grover, L.M and Hukins, D.W.L. Pulsed-lowintensity ultrasound enhances extracellular matrix production by fibroblasts encapsulated in alginate. J Tissue Eng. 2012;3(1) doi: $10.1177 / 2041731412454672$

[9] Khanna, A.; Nelmes, R.T.C.; Gougoulias, N.; Maffulli, N. and Gray, J. The effects of LIPUS on soft-tissue healing: a review of literature. Br Med Bull. 2009;89 (1):169-82.

[10] Ackerman, I. Western Ontario and McMaster Universities Osteoarthritis Index (WOMAC). Aust J Physiother. 2009;55 : 213.

[11] Bellamy, N. The WOMAC Knee and Hip Osteoarthritis Indices: Development, validation, globalization and influence on the development of the AUSCAN Hand Osteoarthritis Indices. Clin Exp Rheumatol. 2005;23(39):148-53.

[12] Paul-Dauphin, A., Guillemin, F., Virion, J. and Briancon, S. 1999. Bias and precision in visual analogue scales: a randomized controlled trial. Am J Epidemiol. 150:1117-27.

[13] Altman, R.; Asch, E.; Bloch, D.; Bole, G.; Borenstein, D.; Brandt, K. and Christy, W. Development of criteria for the classification and reporting of osteoarthritis: classification of osteoarthritis of the knee. Arthritis and rheumatism. 1986;28: 1039-49.
[14] Toledo, S. D.; Trapani, K. and Feldbruegge, E. Rheumatic Diseases. In: Braddom, R.L. (ed). Physical Medicine and Rehabilitation. Edisi ke 4. 2011. Philadelphia, Elsevier Saunders: 769-73.

[15] Aigner, T.; Soder, S.; Gebhard, P.M.; Audrey McAlinden, A. and Haag, J. Mechanisms of Disease: role of chondrocytes in the pathogenesis of osteoarthritis - structure, chaos and senescence. Nature Reviews Rheumatology. 2007;3:391-9. doi: $10.1038 /$ ncprheum0534

[16] Tascioglu, F.; Kuzgun, S.; Armagan, O. and Ogutler, G. Shortterm effectiveness of ultrasound therapy in knee osteoarthritis. The journal of international medical research. 2010;38:1233 42.

[17] Turner, J.A.; Deyo, R.A.; Loeser, J.D.; Von Korff, M. and Fordyce, W.E. The importance of placebo effects in pain treatment and research. JAMA. 1994;271:1609-14

[18] Zubieta, J.K. and Stohler, C.S. Neurobiological mechanisms of placebo responses. Ann NY Acad Sci. 2009.1156:198-210.

[19] Ozgonenel, L.; Aytekin, E. and Durmusoglu, G. A doubleblind trial of clinical effects of therapeutic ultrasound in knee osteoarthritis. Ultrasound Med Biol. 2009;35: 44 - 9.

[20] Robertson, V.J. and Baker, K.G. A review of therapeutic ultrasound: effectiveness studies. Phys Ther. 2001;81:1339-50.

[21] Ingber, D.E. Cellular mechanotransduction: putting all the pieces together again. FASEB J. 2006;20:811-27.

[22] Jia, X.L., Chen, W.Z., Zhou, K. and Wang, Z.B. 2005. Effects of low-intensity pulsed ultrasound in repairing injured articular cartilage. Chin J Traumatol. 8:175-8.

[23] Cook, S.D., Salkeld, S.L., Patron, L.P., Doughty, E.S. and Jones, D.G. 2008. The effect of low-intensity pulsed ultrasound on autologous osteochondral plugs in a canine model. Am J Sports Med. 36:1733-41.

[24] Cook, S.D., Salkeld, S.L., Popich-Patron, L.S., Ryaby, J.P., Jones, D.G. and Barrack, R.L. 2001. Improved cartilage repair after treatment with low-intensity pulsed ultrasound. Clin Orthop Relat Res. 391:S231-43. 